ictal EEG in localizing value. (Harvey AS et al. Epilepsia Sept/Oct 1993; $\underline{34: 869) .}$

Anterior left temporal lobectomy and seizure control had an apparent beneficial effect on illogical thinking in a group of 7 children treated for intractable temporal lobe epilepsy at the University of California at Los Angeles (Caplan R, Shields WD et al. LAm Acad Child Adolesc Psychiatry 1993; 32:604).

\title{
CORPUS CALLOSTOMY FOR INTRACTABLE EPILEPSY
}

The response to corpus callosal section in 64 patients with intractable epilepsy was evaluated in a multicenter study in Australia. Age at time of operation ranged from 3 years to 47 years (mean age 20 years); $43 \%$ underwent callosotomy during childhood, at age $<16$ years. Mean age of onset of epilepsy was 5 years, and mean duration of epilepsy before surgery was 15 years. The most common seizure patterns were generalized tonic-clonic (GTC) (83\%) and drop attacks (73\%). Focal seizures occurred in $42 \%$, of whom half had seizures of frontal lobe origin. The rate of favorable outcome was $60 \%$ for drop attacks, $47 \%$ for tonic seizures, $49 \%$ for GTCs, and $55 \%$ for complex partial seizures. Outcome for drop attacks was more favorable with unilateral seizure activity or generalized slow spike and wave, characteristic of Lennox-Gastaut syndrome; bilateral independent spikes, and severe mental retardation carried a poor prognosis. Improvement followed complete callosotomy in 6 of 10 patients not benefited by anterior callosotomy. (Reutens DC et al. Corpus callosotomy for intractable epilepsy: Seizure outcome and prognostic factors. Epilepsia Sept/Oct 1993;34:904-909). (Reprints: Dr SF Berkovic, Department of Neurology, Austin Hospital, Heidelberg, Victoria, Australia 3084).

COMMENT. In children with medically intractable epilepsy, especially drop attacks of focal or Lennox Gastaut types and CPS of frontal lobe origin, corpus callosotomy should be considered when focal resection is not appropriate. Surgical complications are reported to be mild, and include transient hemiparesis and mutism.

Satisfactory seizure control is reported in $25(74 \%)$ of 34 children following corpus callosotomy performed at the Institute of Neurology, Goiania, Brazil. (Cendes F et al. Epilepsia Sept/Oct 1993;34:910). The majority had mental retardation. Atonic seizures responded best, followed by tonic, GTC, and atypical absence seizures. CPS and myoclonic seizures were not controlled. Of 26 patients with behavior problems, $81 \%$ had a significant decrease in aggressiveness, hyperactivity, and/or attention deficit. Total section was complicated by interhemispheric disconnection symptoms. Mutism, dysarthria, or gait dyspraxia persisted in 5 of 13 patients affected. Section limited to the anterior two thirds was uncomplicated. The neurophysiological basis for corpus callosotomy is the interruption of bilateral ictal synchrony. 